\title{
The Deuteron Beam Polarimetry at Nuclotron-NICA
}

\author{
V. P. Ladygin*, , P. K. Kurilkin*, A. Yu. Isupov*, M. Janek ${ }^{\dagger}$ and S. G. Reznikov* \\ *Joint Institute for Nuclear Research, Dubna 141980, Russian Federation \\ †Physics Department, University of Žilina, Žilina 010 26, Slovak Republic \\ †vladygin@jinr.ru
}

Published 29 February 2016

\begin{abstract}
The current deuteron beam polarimetry at Nuclotron is provided by the Internal Target polarimeter based on the use of the asymmetry in dp- elastic scattering at large angles in the c.m.s. at $270 \mathrm{MeV}$. The calibration of the existing deuteron beam polarimeter at Internal Target in the wide energy range will allow to obtain the accuracy of the vector and tensor beam polarization values of about 3-5\%. Further upgrade of low energy and extracted beam polarimeters is discussed.
\end{abstract}

Keywords: Beam polarization; deuteron; elastic scattering; analyzing powers.

PACS numbers: 29.27.Hj, 24.70+s, 25.40.Hs

\section{Introduction}

The study of the spin structure of two-nucleon and three-nucleon short-range correlations via the measurements of the polarization observables in the deuteron induced reactions is one of the major scientific goals at Nuclotron ${ }^{1-} \cdot 3$ The high precision polarimetry of the deuteron and proton beams is important for these investigations.

Future plans of the spin studies with polarized deuterons at Nuclotron-NICA are related with the construction of new polarized deuteron source. ${ }^{4}$ This source will provide much higher (by the factor of $\sim 10^{3}$ ) and greater variety of the spin modes than POLARIS. ${ }^{5}$ Therefore, the deuteron polarimeters should have a capability to determine simultaneously both vector and tensor components of the beam polarization.

\section{Deuteron Beam Polarimeter at Internal Target}

The polarimeter based on the use of $d p$ - elastic scattering at large angles $\left(\theta_{\mathrm{cm}} \geq 60^{\circ}\right)$ at $270 \mathrm{MeV},{ }^{6}$ where precise data on analyzing powers ${ }^{7-9}$ exist, has been developed

This is an Open Access article published by World Scientific Publishing Company. It is distributed under the terms of the Creative Commons Attribution 3.0 (CC-BY) License. Further distribution of this work is permitted, provided the original work is properly cited. 


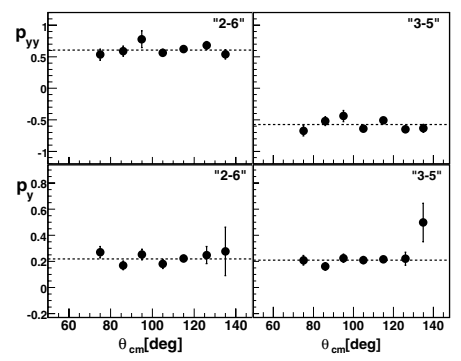

Fig. 1. Tensor $p_{y y}$ and vector $p_{y}$ polarizations of the beam for "2-6" and "3-5" spin modes of POLARIS as a function of the deuteron scattering angle in the c.m.

at internal target station (ITS) at Nuclotron. ${ }^{10}$ The accuracy of the determination of the deuteron beam polarization achieved with this method is better than $2 \%$ because of the values of the analyzing powers were obtained for the polarized deuteron beam, which absolute polarization had been calibrated via the ${ }^{12} \mathrm{C}(d, \alpha){ }^{10} \mathrm{~B}^{*}\left[2^{+}\right]$reaction. ${ }^{9}$

The values of the tensor $p_{y y}$ and vector $p_{y}$ polarizations of the beam for " $2-6$ " and "3-5" spin modes of POLARIS 5 have been measured at several deuteron scattering angle in the c.m. to increase figures of merit as presented in Fig. 1. The estimated figures of merit values for the ITS polarimeter ${ }^{6}$ are comparable with the figures of merit for the deuteron polarimeter used at the extracted beam at RIKEN. ${ }^{9}$

The ITS polarimeter ${ }^{6}$ has been proposed as the reference deuteron polarimeter at Nuclotron-NICA ${ }^{11}$ because it is based on the use of the precise data on the analyzing powers of $d p$ - elastic scattering at $270 \mathrm{MeV}$ obtained at $\mathrm{RARF}^{7-9}$ using the method of the absolute determination of the deuteron beam polarization. ${ }^{9}$ The measurements of $d p$ - elastic scattering performed at $T_{d}$ of $880 \mathrm{MeV}^{12}$ and $2000 \mathrm{MeV}^{13}$ at $\operatorname{ITS}^{10}$ demonstrated that the values of the vector $A_{y}$ and tensor $A_{y y}$ and $A_{x x}$ analyzing powers are large enough to provide the efficient deuteron beam polarimetry at these energies. The $\mathrm{dCH}_{2}$ - quasi-elastic scattering analyzing powers also have large values suitable for the polarimetry. ${ }^{11}$ Therefore, the ITS polarimeter ${ }^{6}$ can be used in the energy range of $300-2000 \mathrm{MeV}$ in the counting mode for the permanent beam polarization monitoring. ${ }^{14}$

\section{Low Energy Deuteron Polarimeter}

Two different low energy polarimeters were used for the measurements of the deuteron beam polarization after LINAC LU-20 for a long period of time. ${ }^{15}$ The first one based on the asymmetry measurement in the $d^{4} \mathrm{He}$ - elastic scattering at backward angles was used for the measurements of the vector component of the beam polarization. The second one utilized the $d^{3} \mathrm{He} \rightarrow p\left(0^{\circ}\right)^{4} \mathrm{He}$ reaction was employed to measure the tensor beam polarization. However, they cannot be used simultaneously to measure both polarization components. 


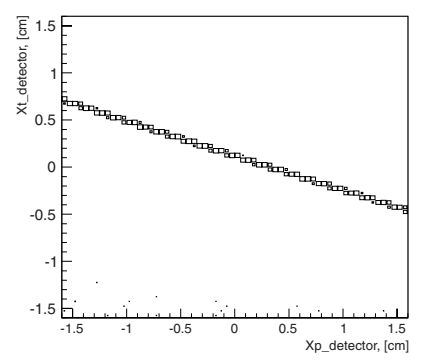

Fig. 2. The correlation of the X-coordinates for protons and tritons registered by the silicon strip detectors.

We propose to design new low energy deuteron polarimeter based on the detection of the charged particles from the $d d \rightarrow{ }^{3} \mathrm{H} p$ reaction at $10 \mathrm{MeV}$. The cross section, deuteron vector and tensor analyzing powers at the scattering angle of $\sim 130^{\circ}$ in the c.m. ${ }^{16}$ are large enough to provide the efficient polarimetry. The thin solid $\mathrm{CD}_{2}$ and carbon targets can be used to obtain the effect on deuterium via $\mathrm{CD}_{2}-\mathrm{C}$ subtraction procedure. This will avoid the use of the gaseous targets. However, the problem of the carbon background elimination appears.

The main option for the detection system is the double sided silicon strip detector developed at LHEP JINR for $\alpha$-particles detection. ${ }^{17}$ The strip pitch is $500 \mu \mathrm{m}$ with the number of strips of 128 in the both $\mathrm{X}$ and $\mathrm{Y}$ directions. The precision of the scattering angle determination in this case will be about 1-2 mrad. The binary reaction $d d \rightarrow{ }^{3} \mathrm{H} p$ will be selected from the other reactions $(d d \rightarrow d d, d d \rightarrow$ pnpn, and $d d \rightarrow d p n$ ) by the proton and triton scattering angles correlation and complanarity condition for the $\mathrm{X}$ - and $\mathrm{Y}$ - coordinates, respectively. These selection criteria will also significantly reduce the contribution from the carbon content of the $\mathrm{CD}_{2}$ target. The additional selection can be done using the energy depositions of the proton and triton in the silicon detectors. Fig. 2 shows simulation results for the correlation of the $\mathrm{X}$ - coordinates for two planes containing the proton and triton detectors. The area inside of the open square in the middle of the Fig. 2 corresponds to the region covered by the both detectors. One can notice that the $d d \rightarrow{ }^{3} \mathrm{H} p$ events can be easily selected from the $d d$ - elastic scattering one and other channels by applying the graphical criteria on the correlation of the $\mathrm{X}$ - and $\mathrm{Y}$ - coordinates for the proton and triton detectors.

\section{High Energy Deuteron Polarimeter}

The tensor-vector deuteron polarimetry at high energies can be provided via using $d p$ - elastic scattering at small angles in the c.m. Both tensor and vector analyzing powers have large values. ${ }^{18,19}$ The feasibility of the $d p$ - elastic scattering events selection using information on the energy losses in the scintillator and timing information has been demonstrated at $\theta_{\text {lab }}^{d} \sim 8^{\circ}$ at the energies 1600 and $2000 \mathrm{MeV} .^{20}$ 


\section{Conclusions}

The reference polarimeter ${ }^{6}$ based on $d p$ - elastic scattering at large angles in the c.m. and placed at ITS $^{10}$ at Nuclotron has been developed. The upgrade of this polarimeter was started, in particular, new control and data acquisition system for ITS $^{21}$ was put into operation.

Low energy polarimeter can be based on the use of the $d d \rightarrow{ }^{3} \mathrm{H} p$ reaction at $10 \mathrm{MeV}$. The development of the the silicon strip detectors to detect protons and tritons in the coincidence was started.

The extracted beam line high energy polarimeters can be based on the use of $d p$ - elastic scattering at small scattering angles in the c.m.. ${ }^{18,19}$

The goal of such deuteron polarimetry scheme is to provide the error in the determination of the polarization components of $3 \%$ at the energies of $270-2000$ $\mathrm{MeV}$ and better than $5 \%$ at higher energies.

\section{Acknowledgments}

The work has been supported in part by the programs of the scientific cooperation between JINR and Slovak Republic in 2014 y. and by the RFBR grant No.13-0200101a.

\section{References}

1. V. P. Ladygin et al., J. Phys. Conf. Ser. 295, 012131 (2011).

2. V. P. Ladygin et al., Phys. Part. Nucl. 45, 327 (2014).

3. V. P. Ladygin et al., Few Body Syst. 55, 709 (2014).

4. V. V. Fimushkin et al., Eur. Phys. J. ST 162, 275 (2008).

5. N. G. Anishchenko et al., AIP Conf. Proc. 95, 445 (1983).

6. P. K. Kurilkin et al., Nucl. Instr. Meth. in Phys. Res. A642, 45 (2011).

7. K. Sekiguchi et al., Phys. Rev. C65, 034003 (2002).

8. K. Sekiguchi et al., Phys. Rev. C70, 014001 (2004).

9. K. Suda et al., Nucl. Instr. Meth. in Phys. Res. A572, 745 (2007).

10. A. I. Malakhov et al., Nucl. Instrum. Meth. in Phys. Res. A440, 320 (2000).

11. P. K. Kurilkin and V. P. Ladygin, Phys. Part. Nucl. 45, 265 (2014).

12. P. K. Kurilkin et al., Phys. Lett. B715, 61 (2012).

13. P. K. Kurilkin et al., Phys. Part. Nucl. Lett. 8, 1081 (2011).

14. T. Uesaka et al., Phys. Part. Nucl. Lett. 3, 305 (2006).

15. Yu. K. Pilipenko et al., AIP Conf. Proc. 570, 801 (2001).

16. W. Grüebler et al., Nucl. Phys. A193, 129 (1972); V. König et al., Nucl.Phys. A331, 1 (1979).

17. A. V. Isaev et al., Instrum. Exp. Tech. 54, 37 (2011).

18. M. Haji-Saied et al., Phys. Rev. C36, 2010 (1987).

19. V. V. Glagolev et al., Eur. Phys. J. A48, 182 (2012).

20. Yu. V. Gurchin et al., Phys. Part. Nucl. Lett. 8, 566 (2011).

21. A. Yu. Isupov et al., Nucl. Instrum. Meth. in Phys. Res. A698, 127 (2013). 